\section{An ultrafast system for signaling mechanical pain in human skin}

\author{
Saad S. Nagi ${ }^{1,2,3 *}{ }^{\dagger}$, Andrew G. Marshall ${ }^{4,5 *}$, Adarsh Makdani ${ }^{5}$, Ewa Jarocka ${ }^{6}$, \\ Jaquette Liljencrantz ${ }^{7,8}$, Mikael Ridderström ${ }^{9}$, Sumaiya Shaikh ${ }^{1,3}$, Francis O'Neill ${ }^{10}$, \\ Dimah Saade ${ }^{11}$, Sandra Donkervoort ${ }^{11}$, A. Reghan Foley ${ }^{11}$, Jan Minde ${ }^{9}$, Mats Trulsson ${ }^{12}$, \\ Jonathan Cole ${ }^{13}$, Carsten G. Bönnemann ${ }^{11}$, Alexander T. Chesler ${ }^{7}$, M. Catherine Bushnell ${ }^{7}$, \\ Francis McGlone $^{5,14}$, Håkan Olausson ${ }^{1,2}$
}

The canonical view is that touch is signaled by fast-conducting, thickly myelinated afferents, whereas pain is signaled by slow-conducting, thinly myelinated ("fast" pain) or unmyelinated ("slow" pain) afferents. While other mammals have thickly myelinated afferents signaling pain (ultrafast nociceptors), these have not been demonstrated in humans. Here, we performed single-unit axonal recordings (microneurography) from cutaneous mechanoreceptive afferents in healthy participants. We identified A-fiber high-threshold mechanoreceptors (A-HTMRs) that were insensitive to gentle touch, encoded noxious skin indentations, and displayed conduction velocities similar to A-fiber low-threshold mechanoreceptors. Intraneural electrical stimulation of single ultrafast A-HTMRs evoked painful percepts. Testing in patients with selective deafferentation revealed impaired pain judgments to graded mechanical stimuli only when thickly myelinated fibers were absent. This function was preserved in patients with a loss-of-function mutation in mechanotransduction channel PIEZO2. These findings demonstrate that human mechanical pain does not require PIEZO2 and can be signaled by fast-conducting, thickly myelinated afferents.

\section{INTRODUCTION}

Cutaneous somatosensory nerve fibers are classified on the basis of their conduction velocity and degree of myelin thickness (1). Rapidly conducting, thickly myelinated cutaneous afferents constitute the $A \beta$ group, whereas slowly conducting, thinly myelinated or unmyelinated afferents belong to the $\mathrm{A} \delta$ and $\mathrm{C}$ groups, respectively $(2,3)$. In humans, all cutaneous $A \beta$ afferents are considered to exclusively signal the discriminative aspects of touch, whereas A $\delta$ and C afferents signal pain ("fast" and "slow" pain, respectively) in addition to temperature and affective touch sensations (4-8). Consistently, the consensus protocol for somatosensory testing in neuropathic pain (9) recommends that the assessment of vibration and punctate touch reflects the functions of $\mathrm{A} \beta$ afferents, whereas the assessment of mechanical pain and temperature reflects the functions of $A \delta$ and $C$ afferents. By implication, this suggests that patients with large-fiber neuropathies have intact pain and temperature perception, whereas

\footnotetext{
${ }^{1}$ Center for Social and Affective Neuroscience, Linköping University, S-581 85 Linköping, Sweden. ${ }^{2}$ Department of Clinical Neurophysiology, Linköping University Hospital, S-581 85 Linköping, Sweden. ${ }^{3}$ School of Medicine, Western Sydney University, Penrith, NSW 2751, Australia. ${ }^{4}$ Faculty of Life Sciences, University of Manchester, M13 9PL Manchester, UK. ${ }^{5}$ School of Natural Sciences and Psychology, Liverpool John Moores University, L3 3AF Liverpool, UK. ${ }^{6}$ Department of Integrative Medical Biology, Umeå University, S-901 87 Umeå, Sweden. ${ }^{7}$ National Center for Complementary and Integrative Health, National Institutes of Health, Bethesda, MD 20892, USA. ${ }^{8}$ Department of Anesthesiology and Intensive Care, Institute of Clinical Sciences, Sahlgrenska Academy, University of Gothenburg, S-413 45 Gothenburg, Sweden. ${ }^{9}$ Department of Surgery, Unit of Orthopedics, Perioperative Sciences, Umeå University Hospital, 90185 Umeå, Sweden. ${ }^{10}$ School of Dentistry, Institute of Clinical Sciences, University of Liverpool, L3 5PS Liverpool, UK. ${ }^{11}$ National Institute of Neurological Disorders and Stroke, National Institutes of Health, Bethesda, MD 20892, USA. ${ }^{12}$ Department of Dental Medicine, Karolinska Institute, S-141 04 Huddinge, Sweden. ${ }^{13}$ Centre of Postgraduate Medical Research and Education, Bournemouth University, Poole BH12 5BB, UK. ${ }^{14}$ Institute of Psychology, Health and Society, University of Liverpool, L3 5DA Liverpool, UK.

*These authors contributed equally to this work.

tCorresponding author. Email: saad.nagi@liu.se
}

patients with small-fiber neuropathies have disturbances in these sensory functions.

The dichotomy into a fast system for touch and a relatively slower system for pain fails to recognize that most other species are equipped with an ultrafast pain system comprising nociceptors in the thickly myelinated $A \beta$ range, the prevalence of which varies from 18 to $65 \%$ of the myelinated nociceptor population across different species (10-13). In the monkey hairy skin, for instance, 12\% of the $\mathrm{A} \beta$ population consists of nociceptors, representing $18 \%$ of the myelinated nociceptor population (12). Further, in humans, highfrequency electrical stimulation of $A \beta$ afferents can generate a painful percept and a nociceptive flexion reflex $(14,15)$.

In mouse hairy skin, $A \beta$ afferents of the field type, a class of rapidly conducting, brush-sensitive mechanoreceptors, "exhibit hallmarks of myelinated nociceptors" (16). Mouse A $\beta$ field afferents display a high threshold for monofilament activation and capacity to encode noxious skin indentations. These are characteristics of a nociceptor according to the definition by the International Association for the Study of Pain: "a high-threshold sensory receptor of the peripheral somatosensory nervous system that is capable of transducing and encoding noxious stimuli" (17). Another recent paper demonstrates that the stretch-gated PIEZO2 ion channel, which is an important receptor for discriminative touch and proprioception (18-20), contributes to mechanical pain signaling in $\mathrm{A} \delta$ and $\mathrm{C}$ afferents in mice (21).

Here, we used the in vivo electrophysiological technique of microneurography (22) to test whether humans have ultrafast highthreshold mechanoreceptive afferents with nociceptive properties. Given the finding in mice (16), it was hypothesized that these afferents might be the field afferents. We also examined rare patients with selective large- and small-fiber neuropathies to test the hypothesis that lack of $A \beta$ afferents affects psychophysical pain judgments to graded mechanical stimuli (henceforth referred to as mechanical pain sensitivity). Last, we examined patients with an inherited loss-of-function (LOF) mutation in the PIEZO2 stretch-gated ion 
channel to test the hypothesis that lack of this channel leads to disturbances in mechanical pain sensitivity.

\section{RESULTS}

Using microneurography (22), we performed single-unit axonal recordings from the peroneal and radial nerves in 103 healthy participants (112 experimental sessions). We recorded from a total of 157 afferents (peroneal, 129; radial, 28), including all five types of A-fiber low-threshold mechanoreceptors (A-LTMRs) as previously defined in human hairy skin (23): field LTMR $(n=66)$, rapidly adapting type 1 (RA1) LTMR (hair unit, $n=18$ ), RA2-LTMR (Pacinian unit, $n=4)$, slowly adapting type 1 (SA1) LTMR $(n=27)$, and SA2-LTMR $(n=19)$. All A-LTMR types responded vigorously to soft brush stroking $(8)$.

\section{High-threshold mechanoreceptors with very fast conduction velocities}

Besides A-LTMRs, we recorded from $18 \mathrm{~A}$-fiber high-threshold mechanoreceptors (A-HTMRs; Fig. 1A), representing 12\% of the A-fiber sample. The receptive field of A-HTMRs comprised multiple small high-sensitivity spots (Fig. 1A). The average size of an A-HTMR receptive field was significantly smaller than the receptive field of field afferents but was not different from that of SA1 afferents (Fig. 1A).
A

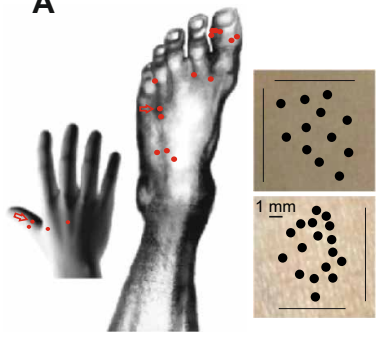

D

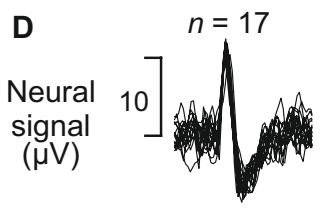

Trigger:

Electrical
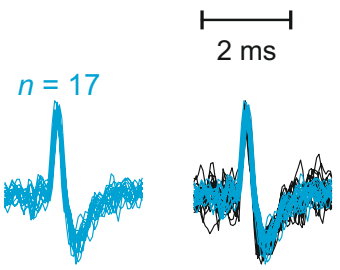

Mechanical
B

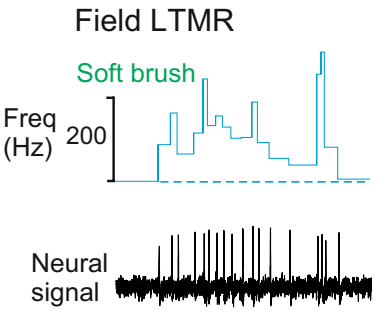

E

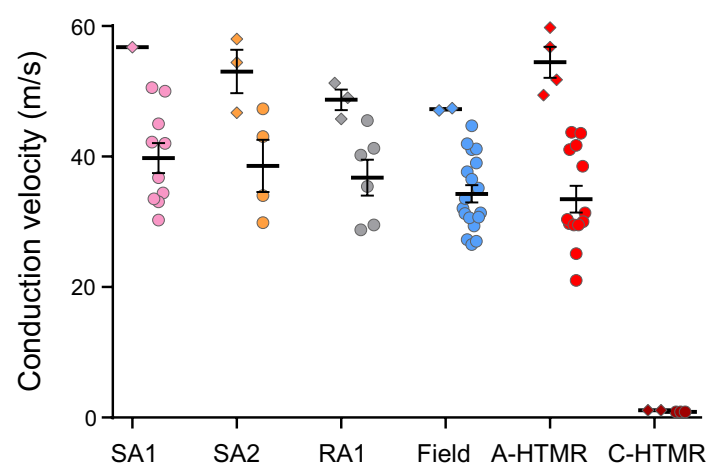

C
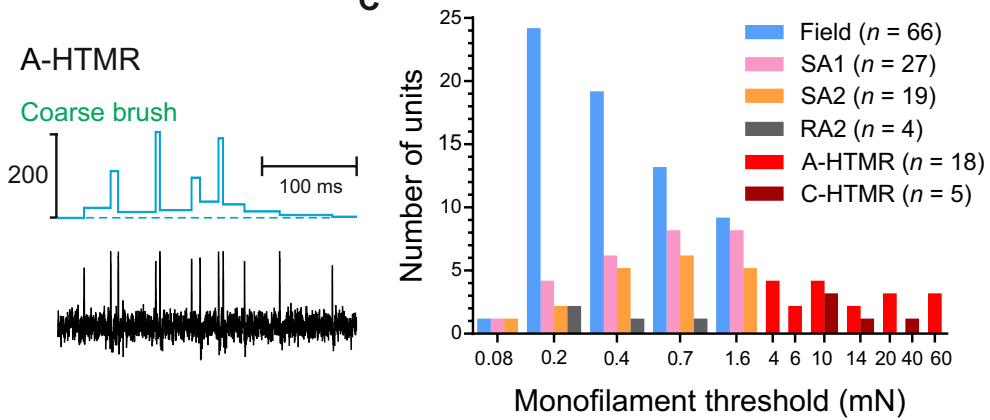

$\mathbf{F}$

Field LTMR

A-HTMR

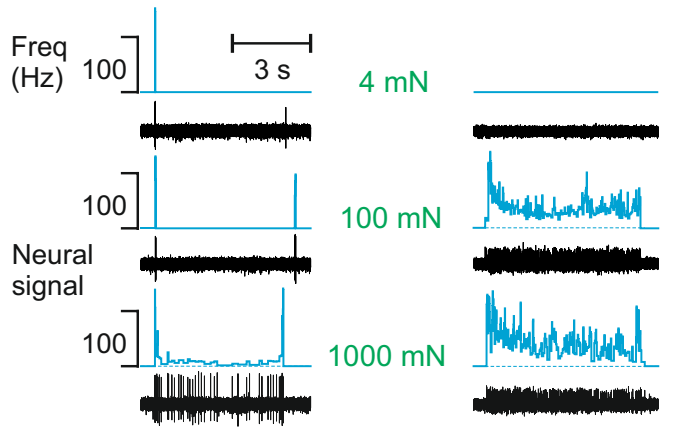

Fig. 1. Humans are equipped with high-threshold and very fast conducting primary afferents. (A) Location of myelinated HTMR receptive fields from recordings in the peroneal and radial nerves. Each red dot represents the location of an individual A-HTMR $(n=18)$. The pattern of receptive field spots, mapped with a Semmes-Weinstein monofilament, is shown for two A-HTMRs (marked by arrows; top, radial; bottom, peroneal). Receptive field spots were redrawn on photographic images so they can easily be seen. The horizontal lines represent the medial-lateral dimension, and the vertical lines represent the proximal-distal dimension. The average size of an A-HTMR receptive field, mapped using a filament force six times higher than that for an A-LTMR, was $26.8 \mathrm{~mm}^{2}( \pm 6.9 ; n=9)$. This was significantly smaller than the receptive field of field afferents $\left(100.5 \pm 7.2 \mathrm{~mm}{ }^{2} ; n=51 ; P<0.0001\right.$, Dunnett's test) but was not different from that of SA1 afferents $\left(41.6 \pm 7.5 \mathrm{~mm}^{2} ; n=17 ; P>0.05\right.$, Dunnett's test). (B) Brush responses of an A-HTMR and a field afferent. Using a soft or a coarse brush, the skin area centered on the receptive field of the recorded afferent was gently stroked at $3 \mathrm{~cm} / \mathrm{s}$. The field afferent responded vigorously to soft brush stroking. The A-HTMR did not respond to soft brush stroking, but it did respond to coarse brush stroking. The mechanical threshold of this A-HTMR was $4 \mathrm{mN}$, and the conduction velocity was $52 \mathrm{~m} / \mathrm{s}$. It responded to pinching, and its receptive field moved with skin translocation. Freq, frequency. (C) Mechanical threshold distribution of HTMRs and LTMRs in the recorded sample. For RA1 afferents, the preferred stimulus is hair movement, so monofilament thresholds were not measured. For A-HTMR, the median mechanical threshold was $10.0 \mathrm{mN}$ $(Q, 5.5-20.0 ; n=18)$. This was significantly higher than the mechanical thresholds of all tested A-LTMR types (at least $P<0.001$ for all individual comparisons, Dunn's test) but was not different from C-HTMRs $(10.0 \mathrm{mN} ; Q, 10.0-27.0 ; n=5)$. (D) Spike activity of an A-HTMR to electrical and mechanical stimulations of the receptive field. Individual electrically and mechanically evoked spikes were superimposed on an expanded time scale to show that the electrically stimulated spike (used for latency measurement) was from the same unit as the one that was mechanically probed at the receptive field. (E) Conduction velocities of HTMRs and LTMRs to surface electrical stimulation (and monofilament tapping in case of one HTMR, conducting at $30 \mathrm{~m} / \mathrm{s}$ ). The data show individual and average ( $\pm \mathrm{SEM}$ ) conduction velocities of single afferents from peroneal (circles) and radial (diamonds) nerves. Conduction velocities of peroneal A-HTMRs $(33.5 \pm 2.1 ; n=13)$ were statistically indistinguishable from peroneal A-LTMRs [SA1:39.8 $\pm 2.3, n=10 ;$;A2: $38.6 \pm 4.0, n=4 ; \mathrm{RA} 1: 36.8 \pm 2.8$, $n=6$; field: $34.3 \pm 1.3, n=18 ; F(4,46)=1.70 ; P=0.17$, one-way analysis of variance (ANOVA)]. All three peroneal C-HTMRs were conducting at $0.9 \mathrm{~m} / \mathrm{s}$. In comparison to the peroneal nerve, conduction velocities of A-fiber types were faster in the radial nerve as expected (A-HTMR: $54.5 \pm 2.4, n=4 ; \mathrm{SA} 1: 56.8 \mathrm{~m} / \mathrm{s}, n=1 ; \mathrm{SA2}: 53.0 \pm 3.3, n=3 ; \mathrm{RA} 1: 48.7 \pm 1.6$, $n=3$; field: $47.3 \pm 0.2, n=2)(46)$. Both radial C-HTMRs were conducting at $1.1 \mathrm{~m} / \mathrm{s}$. Conduction velocity of RA2 afferents was not measured. (F) Slowly adapting properties of an A-HTMR at higher indentation forces. Spike activity of a field afferent and an A-HTMR during monofilament stimulation at three different forces, applied using electronic filaments with force feedback. Compared to the field afferent, the A-HTMR showed a sustained response at a lower indentation force (see also fig. S1). 
All A-HTMRs were insensitive to soft brush stroking; some did not respond at all, and others produced a weak response comprising one to two spikes (one unit was an exception; see Discussion). Further, A-HTMRs showed no response to joint movement but were activated by coarse brush stroking (Fig. 1B). We also recorded from five C-fiber HTMRs (C-HTMRs), confirmed by the 2-Hz electrical stimulation protocol $(24,25)$. These C-HTMRs, akin to A-HTMRs, were insensitive to soft brush stroking. For A-HTMR, the median mechanical threshold was $10.0 \mathrm{mN}$. This was significantly higher than the mechanical thresholds of all tested A-LTMR types but was not different from that of C-HTMRs (Fig. 1C).

On the basis of conduction velocity measurements from 51 peroneal myelinated afferents (Fig. 1, D and E), A-HTMRs were similar to A-LTMRs. A comparison of peroneal with radial $(n=13)$ data revealed that the average conduction velocity of myelinated fibers in the radial nerve was $16.1 \mathrm{~m} / \mathrm{s}$ faster than myelinated fibers in the peroneal nerve. The conduction velocities of the fastest A-HTMR were 43.7 and $59.8 \mathrm{~m} / \mathrm{s}$ in the peroneal and radial samples, respectively.

The A-HTMRs showed rapidly adapting (RA)-like responses at forces near threshold but exhibited slowly adapting (SA)-like responses at higher forces, starting from four to five times threshold (Fig. 1F; for comparison, see fig. S1A for C-HTMR spike activity). The field afferents showed a low-frequency sustained response but at forces that were several hundred times above their threshold (Fig. 1F).

For nine A-HTMRs $(\geq 30 \mathrm{~m} / \mathrm{s})$, we tested force-coding properties. The A-HTMRs encoded force in the perceptibly noxious range of indentation (Fig. 2A), and we found a strong correlation between the A-HTMR peak firing rates and psychophysical pain ratings (Fig. 2, $\mathrm{B}$ and C; see Fig. 2, D to F, for spike numbers and mean firing and, for comparison, see fig. S1, B and C, for C-HTMR responses to graded punctate forces).
A

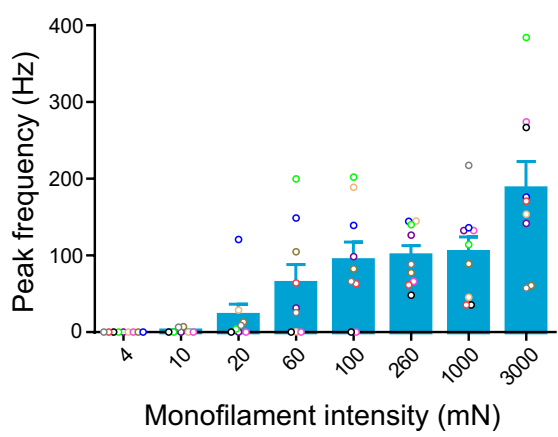

D

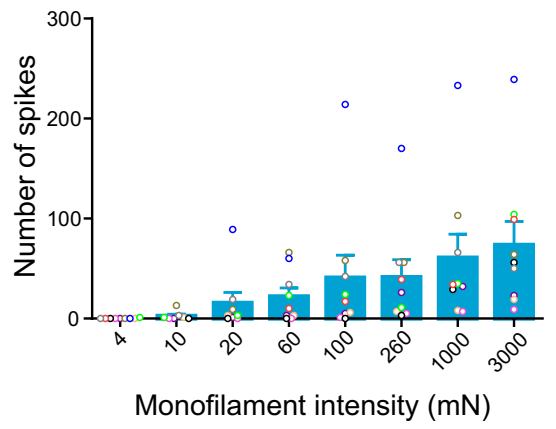

B

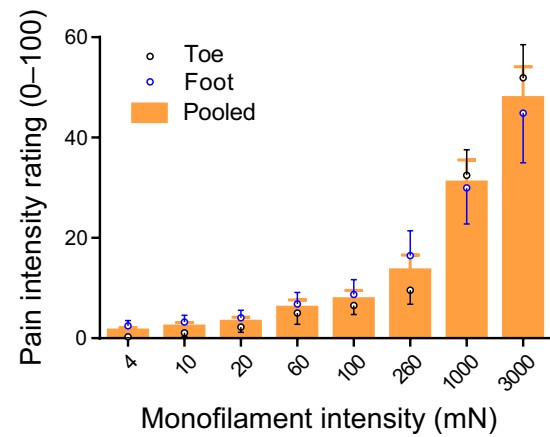

E

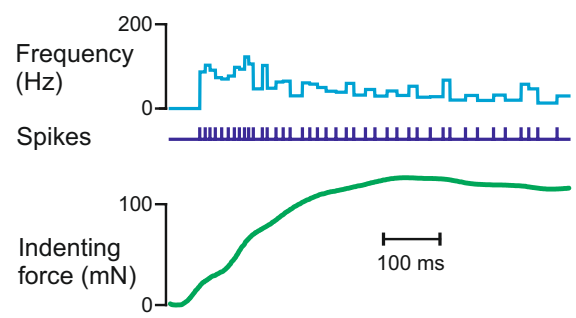

Neural signal

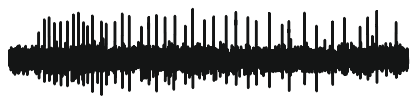

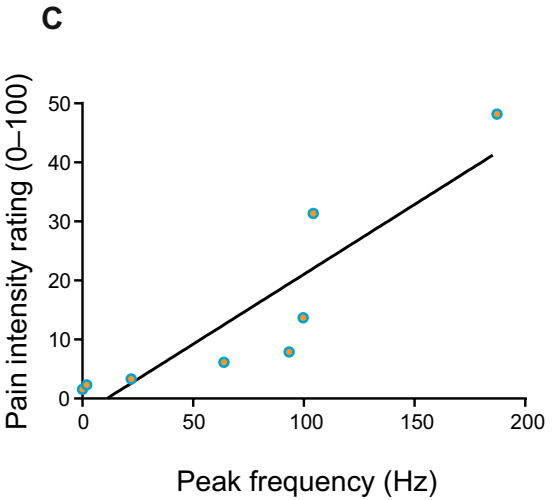

$\mathbf{F}$

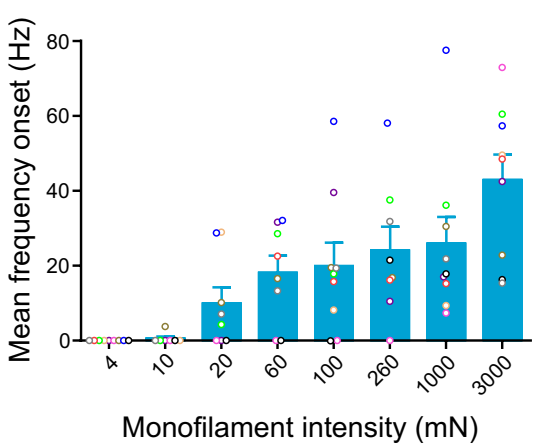

Fig. 2. Neural discharge of A-HTMRs and perception of mechanical pain in response to punctate forces. (A) Peak discharge rates of human A-HTMRs for monofilament stimulation. The data show individual and average $( \pm S E M)$ responses of nine A-HTMRs $(\geq 30 \mathrm{~m} / \mathrm{s})$ to $5-5$ monofilament stimulation at eight different indentation forces. A significant linear fit was displayed $\left(R^{2}=0.6981, P=0.0098\right)$, and the response at the highest indentation force $(3000 \mathrm{mN})$ was significantly higher than responses to all weaker indentation forces (at least $P<0.05$, Dunnett's test). Individual units are color-coded. (B) Psychophysical data for pain intensity to graded monofilament stimulation. Psychophysical pain ratings were collected from 16 healthy participants (dorsal foot: 8 participants, 12 trial sets; dorsal toe: 8 participants, 9 trial sets) from the microneurography sample. The monofilament force had a significant effect on psychophysical pain ratings $[F(8,171)=29.96 ; P<0.0001$, two-way ANOVA], with a significant pain of $\sim 14$ of 100 emerging at $260 \mathrm{mN}$, and higher pain ratings with increasing forces (Tukey's multiple comparisons test). No difference in pain ratings was found between the foot and toe stimulation sites $[F(1,171)=0.1733 ; P=0.6777$, two-way ANOVA]; hence, the data were pooled for a subsequent analysis. (C) Psychophysical pain ratings as a function of neural discharge in A-HTMRs. A comparison of the average peak discharge rates of nine A-HTMRs and average psychophysical pain ratings for skin indentations (eight forces, 4 to $3000 \mathrm{mN}$ ) revealed a significant positive correlation (Pearson $r=0.8944, R^{2}=0.8, P=0.0027$ ). (D) Number of spikes produced by monofilament stimulation in human A-HTMRs. Individual and average $( \pm$ SEM) responses of nine A-HTMRs to 5 -s monofilament stimulation at eight different indentation forces. A significant linear fit was displayed $\left(R^{2}=0.6361, P=0.0177\right)$. Individual units are color-coded. (E) Spike activity of an A-HTMR during the first $0.5 \mathrm{~s}$ of monofilament stimulation (with force feedback). Top: Neural discharge rates. Middle: Spike markers and indentation force markers. Bottom: Neural recording. The first $0.5 \mathrm{~s}$ was selected as the onset period (dynamic phase) of monofilament stimulation (total duration, $5 \mathrm{~s}$ ). (F) Mean discharge rates of human A-HTMRs for monofilament onset. Individual and average $( \pm$ SEM) responses of nine A-HTMRs to the onset period $(0.5 \mathrm{~s})$ of monofilament stimulation at eight different indentation forces. A significant linear fit was displayed $\left(R^{2}=0.6784, P=0.0120\right)$. Individual units are color-coded. 
The responsiveness of human field afferents plateaued as the indentation force increased, and we found no correlation with corresponding psychophysical pain ratings (for details on that and other properties of human field afferents, see fig. S2). Collectively, these observations argue against a nociceptive function for human field afferents.

In two A-HTMRs, thermal sensitivity was tested by applying a computer-controlled thermode to the receptive field (TSA-II, Medoc Ltd.): The temperature changed at $1^{\circ} \mathrm{C} / \mathrm{s}$ from a neutral baseline $\left(32^{\circ} \mathrm{C}\right)$ to painful end points $\left(4^{\circ}\right.$ and $\left.50^{\circ} \mathrm{C}\right)$, but no neural response was evoked. In another A-HTMR, radiant thermal stimulation with no mechanical aspect was tested by holding cold and hot objects just above the receptive field (26): The participant readily perceived them as cold and painfully hot, but no neural response was evoked. We also sought to test responses to hair movement or pulling, but the receptive field was often devoid of any visible hairs; in one case, we shaved the receptive field, but this had no effect on the unit's mechanical sensitivity. In one A-HTMR, we sought to test vibration sensitivity (PIEZO tactile stimulator, Dancer Design, St. Helens, UK): The receptive field itself was not accessible to the vibration probe, so the vibration ( 1 to $500 \mathrm{~Hz}$ ) was applied to an adjacent site, but no response was evoked.

\section{Microstimulation of single A-LTMR and A-HTMR afferents produced different sensations}

Using single-unit intraneural microstimulation $(27,28)$, a technique where the same recording electrode is used to electrically stimulate that same single afferent, a painful percept was reported for A-HTMRs $(6.3 \pm 2.8 \mu \mathrm{A} ; 30 \mathrm{~Hz}$; train durations, 0.5 and $1 \mathrm{~s})$ with a spatial overlap between the physiological receptive field and the perceived area of sensation (projected field) in three microstimulated units from the peroneal nerve, each in separate individuals. For unit 1 (conduction velocity, $43.6 \mathrm{~m} / \mathrm{s}$ ), a "sharp" pain sensation was reported and rated as 80 on a pain scale of 0 to 100 . For unit 2 (conduction velocity, $41.7 \mathrm{~m} / \mathrm{s}$ ), the pain sensation was described as "pinprick" and rated as 20 on a pain scale of 0 to 100 . For unit 3 (conduction velocity, $30.0 \mathrm{~m} / \mathrm{s}$ ), the pain sensation was described as sharp and pinprick, which intensified as the frequency of stimulation was increased (tested up to $300 \mathrm{~Hz}$ ) (27) with no change in the size or location of projected field. Pain ratings were not collected for this afferent.

Notably, microstimulation of single A-LTMRs never produced a painful percept. We observed overlapping receptive and projected fields in $15 \mathrm{~A}$-LTMRs $(6.3 \pm 1.0 \mu \mathrm{A} ; 1$ and $30 \mathrm{~Hz}$; single pulses to train durations of $\leq 1 \mathrm{~s}$ ), producing nonpainful mechanical sensations that were described as "vibrating," "fluttering," and/or "buzzing" for field afferents $(n=10)$; fluttering and vibrating for RA1 $(n=1)$; vibrating for RA2 $(n=1)$; and "pressure" $(n=2)$ or buzzing and vibrating $(n=1)$ for SA1. In two SA1 and an RA1, stimulation frequencies up to $300 \mathrm{~Hz}$ were tested (27), which intensified the pressure or vibratory sensation with no change in the size or location of projected field.

\section{Testing in rare patients revealed a critical role for $A \beta$ afferents, but not PIEZO2 stretch-gated channels, in mechanical pain sensitivity}

Psychophysical data for graded monofilament stimulation were collected in three patient groups with well-defined rare neuropathies and channelopathies. The first group suffered from sensory ganglionopathy that they acquired as adults and resulted in a complete and permanent loss of large fibers with sparing of small fibers (29-31). These patients lacked proprioception and discriminative touch, but their temperature sense was preserved. The second group was selected from a hereditary sensory and autonomic neuropathy cohort type 5 (HSAN-V). This is a congenital disorder involving a mutation of nerve growth factor $\beta$ gene and results in a reduction of small fibers with sparing of large fibers (32-34). Those selected had no temperature perception, but discriminative touch was preserved (see Materials and Methods for other details). The third group also had a congenital disorder involving a PIEZO $2^{\mathrm{LOF}}$ mutation and shared symptoms with the patients with $A \beta$ deafferentation including severe tactile and proprioceptive deficits $(20,35)$.

A comparison of the pain ratings for graded monofilament stimuli (applied to the dorsal foot) revealed significantly lower pain ratings by the patients with $\mathrm{A} \beta$ deafferentation compared to the patients with HSAN-V and healthy control participants. The pain ratings of the patients with HSAN-V and healthy participants were not different from each other (Fig. 3A). Sensory testing on the radial forearm of the patients with PIEZO2 ${ }^{\mathrm{LOF}}$ showed a preserved mechanical pain sensitivity. When the same test was carried out on the forearm of the patients with $A \beta$ deafferentation, no pain emerged at any of the indentation forces (Fig. 3B).

\section{DISCUSSION}

The conduction velocities of the human A-HTMRs were similar to the A-LTMRs, and the properties of human A-HTMRs were in accord with the characteristics of a nociceptor $(13,36)$. Intraneural microstimulation of single A-HTMRs produced a painful sensation having a sharp/pinprick quality, whereas microstimulation of A-LTMRs always produced nonpainful percepts. The importance of this highspeed system for human pain perception was further suggested by reduced pain ratings to noxious punctate stimuli in patients with $\mathrm{A} \beta$ deafferentation and normal pain ratings in patients with $\mathrm{HSAN}-\mathrm{V}$. Last, the findings in patients with PIEZO $2^{\mathrm{LOF}}$ demonstrated that the ability to grade mechanical pain sensations did not require PIEZO2 channels, suggesting that the function of human A-HTMRs is not critically dependent on this ion channel. The existence of these rapidly conducting pain afferents in humans questions the validity of the dichotomous fast touch-slow pain systems in classical teaching, calls for a reappraisal of the neurological views that mechanical pain examination specifically assesses small-fiber function and that painful neuropathies imply small-fiber involvement, and opens novel therapeutic targets in pain disorders.

Contrary to LTMRs, all HTMRs, except one, were insensitive to soft brush stroking. The sole exception in the A-HTMR sample had a relatively low mechanical threshold $(4 \mathrm{mN})$, which was nonetheless higher than all LTMRs, and displayed other nociceptive properties including coding of noxious force and pain sensation to intraneural microstimulation. The same differential effect vis-à-vis soft brush stroking is known for unmyelinated afferents where C-tactile fibers respond vigorously and $\mathrm{C}$ nociceptors are insensitive $(25,37)$.

When comparing the properties of mouse and human field afferents, it is evident that human field afferents do not have nociceptive properties. That the human field afferents, contrary to their mouse namesake (16), have similar mechanical thresholds to other LTMRs and fail to encode skin indentations in the perceptibly noxious (force) range is clear from our current observations, which are based on the largest sample of field unit recordings in humans. 
A

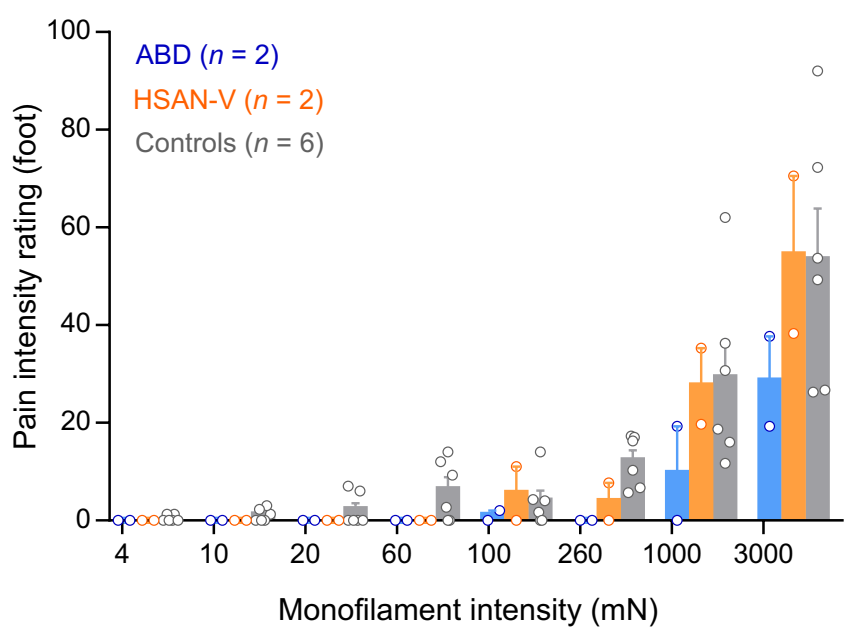

B

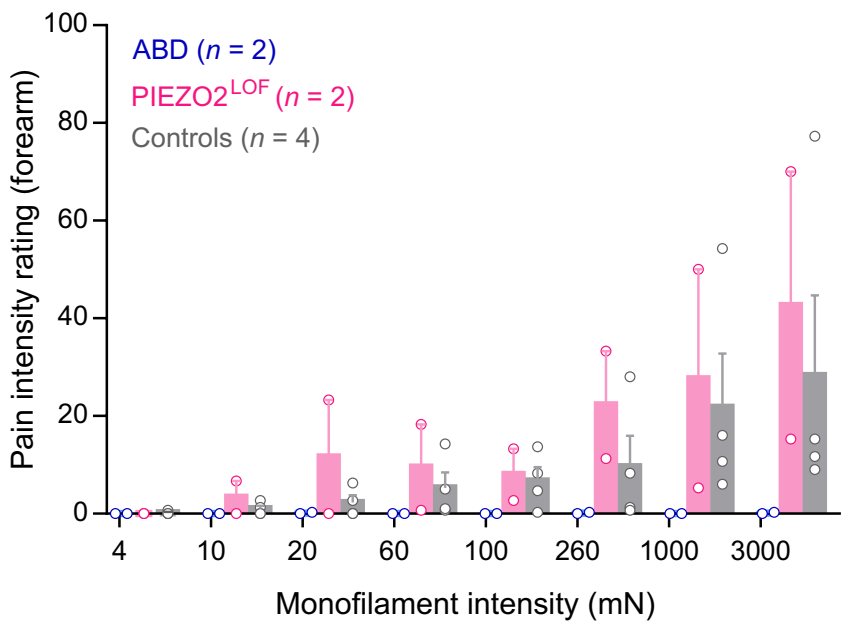

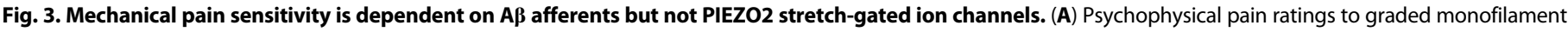

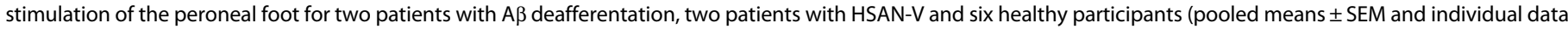

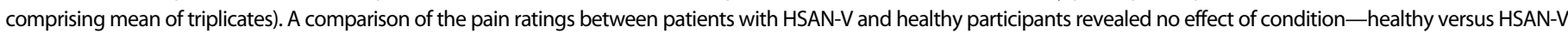

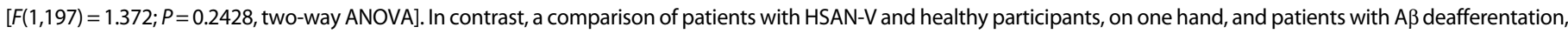

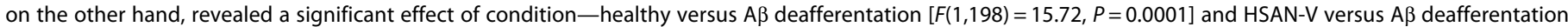

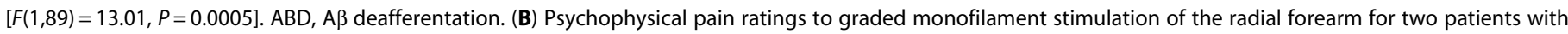

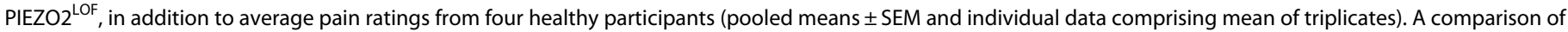

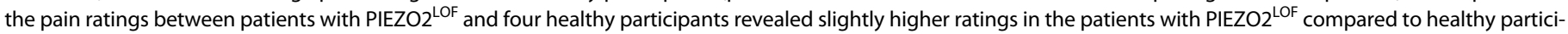

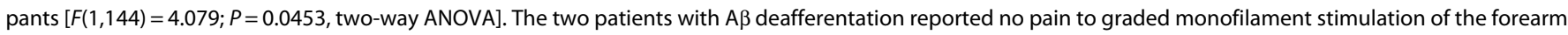

According to conventional theories, a patient with a selective $A \beta$ fiber neuropathy should have intact mechanical pain sensibility because this is widely believed to be a function of the A $\delta$ system. For example, the quantitative sensory testing protocol of the German Research Network on Neuropathic Pain recommends cold detection and mechanical pain testing for the assessment of the $A \delta$ system (9). Yet, the patients with $\mathrm{A} \beta$ deafferentation had normal cold detection but abnormal mechanical pain perception, suggesting that the mechanical pain deficit was related to the loss of $A \beta$ fibers. However, this need not preclude the role of A $\delta$ mechano-nociceptors, and it is unclear whether normal cold thresholds exclude impairment of $A \delta$ mechano-nociceptors in these patients. In contrast to patients with $\mathrm{A} \beta$ deafferentation, a subset of patients with HSAN-V had abnormal thermal detection, but mechanical pain perception was normal. These findings suggest that mechanical pain is not exclusively signaled by the small-fiber system, with the clinically important implication being the decoupling of mechanical pain from the $A \delta$-mediated cold perception and the possibility of perturbed pain perception in A $\beta$ fiber neuropathies.

A recent mouse study (21) showed that PIEZO2 channels contribute to mechanical nociceptive signaling in $\mathrm{A} \delta$ and $\mathrm{C}$ fibers. However, in three patients with PIEZO2 ${ }^{\mathrm{LOF}}$ mutation, mechanical pain thresholds were within normal range (35). Here, we were not able to demonstrate any disturbance in pain judgments to graded mechanical stimuli in two patients with PIEZO2 ${ }^{\mathrm{LOF}}$ mutation. However, PIEZO2 may contribute to aspects of human pain perception that were not captured by our testing protocol. Together, the emerging picture is that mechanical pain in humans is subserved by A-HTMRs but not critically dependent on PIEZO2.

Perhaps, the reason ultrafast nociceptors have received less attention in the animal literature (and have been almost entirely ignored in human work) is that their proportion relative to the overall myelinated nociceptor population decreases from rodents to primates (10-13). Another reason is that the thickly myelinated LTMRs likely dominate, by sheer numbers, the wave of the compound action potential $(10,38)$.

Thickly myelinated nerve fibers are well suited for execution of withdrawal reflexes, given the short latency of signal transmission, as indeed was postulated almost 40 years ago $(14,15)$. In comparison to C-HTMRs, the A-HTMRs displayed a higher firing rate, a more finely grained receptive field with multiple small high-sensitivity spots, and less propensity for fatigue during sustained noxious mechanical stimulation (tested up to $1 \mathrm{~min}$ ). These characteristics likely ensure a large, continuous transfer of nociceptive information to the central nervous system with content rich on stimulus quality, localization, and such - features that need not be limited to the ultrafast nociceptors but may apply to varying extents to other types of myelinated nociceptors (39-43).

The classification of primary afferents into $A \beta$ and $A \delta$ groups based on conduction velocity is not clear-cut in humans. Therefore, we did not classify the myelinated afferents as $A \beta$ or $A \delta$ in the current study. It is suggested that the "upper border of the main part of the D hair CV [conduction velocity] range" provides "a good indication of the upper end of the A $\delta$ CV range" (10). The limitation of this, however, is in its extrapolation to humans where no detailed account of D hair units exists (39).

In electrophysiological investigations in the monkey, there are two distinct peaks in the conduction velocity distribution (15 and $43 \mathrm{~m} / \mathrm{s}$ ), and the $A \beta / A \delta$ boundary is set "at the minimum between these two peaks, which is $30 \mathrm{~m} / \mathrm{s}$ " (12). Applying the same $A \beta / A \delta$ boundary to our sample revealed $15 \mathrm{~A} \beta$ HTMRs with conduction velocity of $\geq 30 \mathrm{~m} / \mathrm{s}$, representing $88 \%$ of the sample of A-HTMRs 
where conduction velocities were measured (all except one unit). This proportion is higher than that reported for other species $(10-13)$ and likely reflects a sampling bias of our study rather than the true proportion of ultrafast nociceptors in humans. In another microneurography study (39), a preponderance of slowly conducting afferents (conduction velocity, $<30 \mathrm{~m} / \mathrm{s}$ ) was reported in the A-HTMR sample from the radial nerve with the fastest A-HTMR signaling at $33 \mathrm{~m} / \mathrm{s}$.

Single-afferent conduction velocity measurements tend to be lower than those obtained by electrical stimulation of the nerve trunk. Factors such as axonal tapering, branch points, and mechanical/ electrical coupling time are thought to be involved $(44,45)$. The conduction velocities in our sample, measured using electrical stimulation (or mechanical in one case) at the receptive field, are consistent with the data from other studies [e.g., (45)]. Notably, the A-HTMRs and A-LTMRs in our sample had similar conduction velocities. As expected (46), conduction velocities were slower in the lower limb than in the upper limb.

In the current study, we recorded from the cutaneous afferents of the peroneal and radial nerves, but it may be that a high-speed system for mechanical pain signaling exists in deep somatic tissue as well. For instance, in peroneal nerve recordings from human muscle nociceptors (47), a "Group II" nociceptor produced "sharp pain" to intraneural microstimulation with its receptive field located in a tendon at the base of the big toe. This unit had a conduction velocity of $32 \mathrm{~m} / \mathrm{s}$; in comparison, the fastest Group III afferent in the sample had a conduction velocity of $13.5 \mathrm{~m} / \mathrm{s}$ (mean, $6.7 \mathrm{~m} / \mathrm{s}$ ).

The average $A \delta$ propagation velocities in humans as studied using laser- or "epidermal electrical"-evoked potentials are typically in the range of 10 to $15 \mathrm{~m} / \mathrm{s}(48,49)$. The latencies of pinprick-evoked potentials are shorter than laser-evoked potentials, thus suggesting a possible contribution of ultrafast A-HTMRs to pinprick-evoked potentials and augmentation thereof (50) in a sensitized state. Further, in the current study, we have shown that electrical activation of ultrafast A-HTMRs (single-unit microstimulation) can evoke a painful percept having a sharp/pinprick quality. We did not track the temporal profile of pain sensation during single A-HTMR microstimulation, e.g., whether it outlasts the stimulus duration, but we aim to explore the resulting perception in more detail in further studies. However, the efficacy of pain therapies based on the gate control theory (51) such as transcutaneous electrical nerve stimulation-a technique using brief, low-intensity electrical pulses to activate A $\beta$ afferents for pain relief-would suggest that the net effect of stimulation of a population of $A \beta$ afferents is not nociceptive [see also (52)]. At the spinal level, at least in mice, fast-conducting cutaneous A-LTMRs project exclusively to deeper laminae of the dorsal horn, whereas fast-conducting cutaneous A-HTMRs project to deeper and superficial laminae (53). The spinal projections of human A-HTMRs remain to be studied.

Slowly conducting, myelinated nociceptors in the human radial nerve (39) show a greater specificity for mechanical stimulation relative to unmyelinated nociceptors, which are often polymodal, an observation that is consistent with other species $(13,36)$. In the mouse, for instance, there are myelinated nociceptors that respond specifically to high-threshold mechanical stimulation (41) including hair pulling (40); in our sample, the receptive fields of A-HTMRs lacked visible hairs, and we were thus not able to test whether they responded to hair pulling. By implication, the ultrafast A-HTMRs in humans may be highly specific for mechanical stimulation (pure mechanonociceptors), and we have no observation to the contrary. However, the human A-HTMR population may consist of subpopulations distinguished not only by differences in conduction velocities but also by their response properties. This needs to be systematically tested in a larger population.

\section{CONCLUSIONS}

We have identified human nociceptors with conduction velocities similar to touch afferents. The ultrafast pain system exists in other mammals, but its existence in humans had hitherto remained a mystery. Further investigations are warranted into their detailed response properties, ion channel properties, and spinal and cortical projections, given the implications for understanding nociception, nocifensive behavior, and clinical pain states.

\section{MATERIALS AND METHODS}

Single-unit axonal recordings (microneurography) were performed from the left peroneal nerve or the right radial nerve of 103 healthy participants (71 males and 32 females; 20 to 49 years), with some of them recruited more than once with an interval of at least 3 months (112 experimental sessions in total). In 16 participants from the microneurography sample, psychophysical testing for mechanical pain was performed. In addition, psychophysical testing for mechanical pain and thermal testing were performed in two patients with A $\beta$ deafferentation (IW: male, 64 years; GL: female, 69 years) (29-31), two patients with HSAN-V (both males, 74 and 86 years) (32-34), and six controls (five females and one male; 55 to 67 years). For HSAN-V, a cohort of 23 patients ( 12 males and 11 females; 25 to 86 years) with confirmed diagnosis were screened for temperature perception, and those with severely diminished temperature perception were selected. Psychophysical testing for mechanical pain was also performed in two patients with PIEZO2 ${ }^{\mathrm{LOF}}$ (female, 12 years; male, 16 years) (20) and four controls (three males and one female; 20 to 23 years).

All adult participants, as well as guardians of patients with PIEZO $2^{\mathrm{LOF}}$, provided informed consent in writing before the start of the experiment. The study was approved by the ethics committees of Linköping University (dnr 2015/305-31), Liverpool John Moores University (14/NSP/039), and National Institutes of Health's Combined Neuroscience Ethics Committee (16-AT-0077) and complied with the revised Declaration of Helsinki.

All participants were seated on a chair with legs or arms stretched out (and hand pronated for the radial nerve) and supported with vacuum pillows. Care was taken to ensure that each participant was comfortably seated and well adjusted to room temperature $\left(22^{\circ} \mathrm{C}\right)$ before starting the experiment. If subjectively cold, then the participants were covered in a blanket except for the test region. Two microneurography setups were used, henceforth referred to as setups 1 and 2 .

\section{Microneurography}

The course of the peroneal nerve at the level of the knee was approximated by surface electrical stimulation ( 1 to $4 \mathrm{~mA}, 0.2 \mathrm{~ms}, 1 \mathrm{~Hz}$ ) using a 2-mm-diameter probe connected to an optically isolated constant-current stimulator (setup 1: FE180 Stimulus Isolator, ADInstruments, Oxford, UK). For radial nerve experiments, the course of the nerve just above the elbow was visualized using ultrasound (LOGIQ e, GE Healthcare, Chicago, IL, USA). A high-impedance tungsten recording electrode was inserted percutaneously, followed 
by precise localization of the nerve by delivery of weak electrical pulses ( 0.02 to $1 \mathrm{~mA}, 0.2 \mathrm{~ms}, 1 \mathrm{~Hz}$; FHC Inc., Bowdoin, ME, USA). The electrode was insulated, except for the $\sim 5-\mu \mathrm{m}$ bare tip, with a typical length of $20 \mathrm{~mm}$ for the peroneal nerve and $40 \mathrm{~mm}$ for the radial nerve (shaft diameter, $0.2 \mathrm{~mm}$ ). An uninsulated subdermal electrode in an adjacent area served as the reference. A high-impedance preamplifier was taped to the skin near the recording electrode (setup 1: MLT185 headstage, ADInstruments, Oxford, UK), which was used in conjunction with a low-noise high-gain amplifier (setup 1: FE185 Neuro Amp EX, ADInstruments, Oxford, UK). Once the electrode tip was intrafascicular, indicated by the subject's reports of cutaneous sensations to weak electrical stimulation $(\leq 0.02 \mathrm{~mA})$, the neural activity was amplified. Single LTMRs were searched for by soft brush stroking ( 1 to $10 \mathrm{~cm} / \mathrm{s}$ ), and single HTMRs were searched for by coarse brush stroking ( 1 to $10 \mathrm{~cm} / \mathrm{s}$ ) or pinching in the fascicular innervation zone while making small adjustments to the electrode position (25). A soft goat hair brush and a coarse synthetic brush with a bristle length of $30 \mathrm{~mm}$ each were used for soft and coarse brush stroking, respectively. A subset of experiments was performed using setup 2 in which the data were digitally sampled using SC/ ZOOM developed by the Physiology Section, Department of Integrative Medical Biology, Umeå University.

\section{Unit identification}

Individual A-LTMRs (all soft brush sensitive) were separated into RA and SA types on the basis of their adaptive responses to ramp-andhold indentation of the skin, as per the criteria used in Vallbo et al. (23). Three groups of RA units were identified as follows: A $\beta$ RA1-LTMR (hair unit), responsive to deflection of individual hairs and light air puffs-air puff responses were abolished after hair removal; $A \beta$ field LTMR, comprising multiple spots of high sensitivity with no response to hair displacement or remote tapping of the skin; and A $\beta$ RA2-LTMR (Pacinian unit), comprising a single spot of maximal sensitivity and robust response to remote tapping/ vibration. Two groups of SA-LTMRs (types 1 and 2) were identified where several features were examined including spontaneous firing, stretch sensitivity, and receptive field characteristics. In addition, an interspike interval pattern to sustained indentation $(100 \mathrm{mN}$ for 30 to $60 \mathrm{~s}$ ) was tested, where possible, with a skewed and broad pattern for SA1 and a normal distribution for SA2. Coefficients of variation of interspike intervals $(23,54)$ were 0.80 for SA1 (median; range, 0.32 to $3.53 ; n=18$ ) and 0.29 for SA2 (median; range, 0.15 to 0.60 ; $n=10)$.

Mechanical threshold and receptive field size of individual units were determined using Semmes-Weinstein monofilaments (nylon fiber; Aesthesio, Bioseb, Pinellas Park, FL, USA). If a unit responded to the same (weakest) monofilament in at least $50 \%$ of trials, then it was taken as the mechanical threshold. Measurement of mechanical threshold was the minimum criterion for inclusion in the sample for all LTMR types, except RA1-LTMRs whose preferred stimulus is hair movement, and thus, responsiveness to light air puffs was determined. For receptive field mapping, predetermined monofilament forces of 100 and $600 \mathrm{mN}$ were used for LTMRs and HTMRs, respectively. The receptive field area was estimated by treating it as an ellipse. Latency responses to surface electrical stimulation of the receptive field were captured, which, together with the distance from the stimulation site to the recording electrode, were used to calculate the conduction velocity (in meters per second). Electrically and mechanically evoked spikes were compared on an expanded time scale to ensure that the electrically stimulated unit was the same as the one that was mechanically probed (Fig. 1D). In one instance, the response latency was estimated by rapid mechanical tapping, using an electronic filament, of a receptive field spot.

\section{Mechanical stimulation}

The punctate force-coding function of individual units was tested with a range of forces, applied to high-sensitivity spots in the receptive field, using commercially available monofilaments (as mentioned earlier), in addition to custom-built electronic monofilaments that allowed high-resolution (1 ms) force measurements using capacitive sensors (nylon fiber, Physiology Section, Department of Integrative Medical Biology, Umeå University). Each monofilament trial set comprised eight consecutive applications of different forces, applied for 5 s each: 4, 10, 20, 60, 100, 260, 1000, and $3000 \mathrm{mN}$ (corresponding values in pressure, 16, 24, 27, 53, 68, 106, 193, and $292 \mathrm{~g} / \mathrm{mm}^{2}$ ). The trial commenced when the filament came into contact with the skin and lasted until it was withdrawn. When using the commercial apparatus, the start and end of the filament-skin contact were indicated with a push button by the experimenter. Where the recording was stable, data for replicate trials of each monofilament force were collected.

\section{Single-unit intraneural microstimulation}

The same recording electrode was also used to stimulate that same axon using low-current electrical pulses (frequencies, $30 \mathrm{~Hz}$ to start off; range, 1 to $300 \mathrm{~Hz}$; pulse width, $0.2 \mathrm{~ms}$; intensity (in a microampere range), starting from zero in small increments until a sensation was reported) $(27,55)$. Stimuli were delivered as single pulses or as trains lasting 0.25 to $1 \mathrm{~s}$. That the same recorded afferent was electrically stimulated depends on the spatial overlap of the evoked percept, i.e., the projected field, with the physiological receptive field $(27,28)$. This was determined by questioning the participant about the location of the projected field relative to the location of mechanical touch (56). Where a receptive field-projected field overlap was found, sensory qualities of the electrically evoked sensation were noted by asking the participant to describe what they felt; this was followed by a structured questionnaire (28).

\section{Psychophysics}

In 16 healthy participants from the microneurography sample, psychophysical pain ratings to graded monofilament stimulation of the dorsal foot were collected (dorsal foot, $n=8$; dorsal toe, $n=8$ ). Eight monofilament forces were applied for $5 \mathrm{~s}$ each, as used in the neural recordings, in random order (three repeats of each force) guided by a custom-written script in MATLAB (R2014b, The MathWorks Inc., Natick, MA, USA). While performing this task, the participants were prevented from viewing the stimuli. Participants were asked to rate the pain intensity on a computerized visual analog scale, ranging from 0 (no pain) to 100 (most intense pain), using the arrow keys of a computer keyboard.

To test whether human $A \beta$ afferents contribute to pain perception and whether this function is dependent on the stretch-gated ion channel PIEZO2, we collected psychophysical data for graded monofilament stimulation in patients with selective deafferentation and those with PIEZO $2^{\mathrm{LOF}}$ mutation. The same monofilament protocol was followed in patients as in healthy participants. The only exception was that the patients with $A \beta$ deafferentation could not operate the keyboard themselves - to rate pain intensity on a computerized 
visual analog scale-because of a complete loss of proprioception. Therefore, they were asked to point at the screen, and the experimenter pressed the keyboard keys accordingly. Patients with PIEZO2 ${ }^{\mathrm{LOF}}$ also had proprioceptive deficits $(20,35)$, but they could operate the keyboard. Psychophysical testing in patients with $A \beta$ deafferentation

IW and GL are well-characterized patients with $A \beta$ deafferentation who suffer from a rare sensory neuronopathy syndrome-a disorder that selectively affects large primary sensory neurons' cell bodies in the dorsal root ganglia (57)-resulting in a complete and permanent loss of $\mathrm{A} \beta$ afferent fibers below $\mathrm{C} 3$. However, the cold sensing A $\delta$ system, which is also thought to be the substrate for mechanical pain (9), is intact, as documented in previous clinical and neurophysiological studies (29-31). At the start of the experiment, we measured the cold detection threshold, a function of A $\delta$ system, at the dorsal foot/distal leg in triplicates (TSA-II, Medoc Ltd., Ramat Yishai, Israel) and found it to be normal in patients with $\mathrm{A} \beta$ deafferentation [means \pm SEM, $26.2^{\circ} \pm 0.6^{\circ} \mathrm{C}$; controls, $27.0^{\circ} \pm 0.6^{\circ} \mathrm{C}(n=6)$; baseline $=32.0^{\circ} \mathrm{C}$ ]. Cold detection was also tested at the dorsal forearm, and the threshold $\left(27.4^{\circ} \pm 0.5^{\circ} \mathrm{C}\right)$ was similar to the foot region. In addition to triplicate trials, a more extensive set of 10 cooling trials (interspersed with warming trials) was tested in GL. The detection threshold for the 10 trials was $27.0^{\circ} \pm 0.5^{\circ} \mathrm{C}$.

\section{Psychophysical testing in patients with HSAN-V}

A mutation in the nerve growth factor $\beta$ gene, classified as HSAN-V, results in a reduction of $A \delta$ and $C$ fibers with sparing of $A \beta$ fibers, as documented in previous clinical and neurophysiological tests including nerve biopsies and conduction studies (32-34). In this wellcharacterized cohort $(n=23)$, who were heterozygous for the mutation, thermal and thermal pain testing (dorsal foot; triplicate trials; test range, $20^{\circ}$ to $\left.50^{\circ} \mathrm{C}\right)$ revealed two subjects who had no cold $\left(<20^{\circ} \mathrm{C}\right)$ or heat pain $\left(>50^{\circ} \mathrm{C}\right)$ perception, with warm only reported at noxious temperatures $\left(47.5^{\circ} \pm 0.9^{\circ} \mathrm{C}\right)$. The corresponding values for controls $(n=6)$ were $27.0^{\circ} \pm 0.6^{\circ} \mathrm{C}$ (cold detection), $39.7^{\circ} \pm 0.7^{\circ} \mathrm{C}$ (warm detection), and $44.0^{\circ} \pm 0.7^{\circ} \mathrm{C}$ (heat pain threshold). However, HSAN-V subjects were able to detect vibration or focal touch, tested bilaterally at the great toe using a $128-\mathrm{Hz}$ tuning fork or a $100-\mathrm{mN}$ monofilament, as per the protocol defined in the Michigan Neuropathy Screening Instrument (58). Further, sural nerve conduction test was performed in one of them and was within the normal range.

\section{Psychophysical testing in patients with PIEZO2 $2^{\text {LOF }}$}

In patients with $\mathrm{PIEZO} 2^{\mathrm{LOF}}$ mutation, who had severe proprioceptive and tactile deficits and normal mechanical pain thresholds $(20,35)$, graded monofilament stimuli were tested for their ability to grade the intensity of mechanical pain. The patients had a history of foot deformities and surgical corrective procedures; thus, testing was preferred in the upper limb (radial forearm). Mechanical pain sensitivity was initially tested in three patients with PIEZO2 ${ }^{\mathrm{LOF}}$, but one of them repeatedly fell asleep during the experiment so data could not be reliably collected on him.

\section{Data collection, processing, and analysis}

For microneurography setup 1, the neural activity was sampled at $20 \mathrm{kHz}$, and all data were recorded and processed using the data acquisition and analysis program (LabChart Pro software v8.1.5 and PowerLab 16/35 hardware PL3516/P, ADInstruments, Oxford, UK). Threshold crossing was used to distinguish action potentials from background noise with a signal-to-noise ratio of at least 2:1, followed by template matching to confirm spike morphology.
For microneurography setup 2, the neural signal and forces recorded from the electronic monofilaments were digitally acquired at 19.2 and $1.2 \mathrm{kHz}$, respectively, using SC/ZOOM (Physiology Section, Department of Integrative Medical Biology, Umeå University). Single action potentials were identified semiautomatically under visual control (59). Before any further data processing, recordings were discarded if the analyses indicated the presence of multiple units or if the signal-to-noise ratio prevented secure action potential identification.

The instantaneous discharge rates were generated as time series with values corresponding to the inverse of interspike intervals. For setup 2, force signals, timing of individual spikes, and the instantaneous discharge rate were exported at $1 \mathrm{kHz}$ from SC/ZOOM to MATLAB (R2014b, The MathWorks Inc., Natick, MA, USA).

For monofilament stimulation, the 500-ms window following the first spike was defined as the trial onset, and similarly, the offset of a trial was defined as the 500 -ms window preceding the last spike. In addition, the total period (onset-hold-offset) from the first to the last spike was also analyzed.

For each trial, the number of spikes was counted. The corresponding mean frequency was defined as the number of interspike intervals divided by the sum of the interspike interval duration, and the peak frequency was defined as the maximum value of the instantaneous frequency during the period.

All data were entered in GraphPad Prism for statistical comparisons (version 6.07, GraphPad Software Inc. La Jolla, CA, USA). The type of statistical test used for each comparison is described alongside all statistical data. Figures were generated using CorelDRAW (X8, version 18.1.0.661, Corel Corporation, Ottawa, ON, Canada).

\section{SUPPLEMENTARY MATERIALS}

Supplementary material for this article is available at http://advances.sciencemag.org/cgi/ content/full/5/7/eaaw1297/DC1

Fig. S1. Response properties of C-HTMRs to graded mechanical stimuli.

Fig. S2. Human field afferents do not display nociceptive properties.

\section{REFERENCES AND NOTES}

1. D. P. C. Lloyd, Neuron patterns controlling transmission of ipsilateral hind limb reflexes in cat. J. Neurophysiol. 6, 293-315 (1943).

2. E. D. Adrian, Y. Zotterman, The impulses produced by sensory nerve endings: Part 3. Impulses set up by touch and pressure. J. Physiol. 61, 465-483 (1926).

3. E. D. Adrian, The impulses produced by sensory nerve-endings: Part 4. Impulses from pain receptors. J. Physiol. 62, 33-51 (1926).

4. H. E. Torebjörk, R. G. Hallin, Perceptual changes accompanying controlled preferential blocking of $\mathrm{A}$ and $\mathrm{C}$ fibre responses in intact human skin nerves. Exp. Brain Res. 16, 321-332 (1973).

5. R. A. Mackenzie, D. Burke, N. F. Skuse, A. K. Lethlean, Fibre function and perception during cutaneous nerve block. J. Neurol. Neurosurg. Psychiatry 38, 865-873 (1975).

6. J. Ochoa, E. Torebjörk, Sensations evoked by intraneural microstimulation of $C$ nociceptor fibres in human skin nerves. J. Physiol. 415, 583-599 (1989).

7. B. Bromm, R. D. Treede, Nerve fibre discharges, cerebral potentials and sensations induced by $\mathrm{CO}_{2}$ laser stimulation. Hum. Neurobiol. 3, 33-40 (1984).

8. L. S. Löken, J. Wessberg, I. Morrison, F. McGlone, H. Olausson, Coding of pleasant touch by unmyelinated afferents in humans. Nat. Neurosci. 12, 547-548 (2009).

9. R. Rolke, R. Baron, C. Maier, T. R. Tölle, R. D. Treede, A. Beyer, A. Binder, N. Birbaumer, F. Birklein, I. C. Bötefür, S. Braune, H. Flor, V. Huge, R. Klug, G. B. Landwehrmeyer, W. Magerl, C. Maihöfner, C. Rolko, C. Schaub, A. Scherens, T. Sprenger, M. Valet, B. Wasserka, Quantitative sensory testing in the German Research Network on Neuropathic Pain (DFNS): Standardized protocol and reference values. Pain 123, 231-243 (2006).

10. L. Djouhri, S. N. Lawson, A $\beta$-fiber nociceptive primary afferent neurons: A review of incidence and properties in relation to other afferent A-fiber neurons in mammals. Brain Res. Rev. 46, 131-145 (2004). 
11. M. Koltzenburg, C. L. Stucky, G. R. Lewin, Receptive properties of mouse sensory neurons innervating hairy skin. J. Neurophysiol. 78, 1841-1850 (1997).

12. R.-D. Treede, R. A. Meyer, J. N. Campbell, Myelinated mechanically insensitive afferents from monkey hairy skin: Heat-response properties. J. Neurophysiol. 80, 1082-1093 (1998).

13. P. R. Burgess, E. R. Perl, Myelinated afferent fibres responding specifically to noxious stimulation of the skin. J. Physiol. 190, 541-562 (1967).

14. J. C. Willer, F. Boureau, D. Albe-Fessard, Role of large diameter cutaneous afferents in transmission of nociceptive messages: Electrophysiological study in man. Brain Res. 152, 358-364 (1978).

15. J.-C. Willer, D. Albe-Fessard, Further studies on the role of afferent input from relatively large diameter fibers in transmission of nociceptive messages in humans. Brain Res. 278 318-321 (1983).

16. L. Bai, B. P. Lehnert, J. Liu, N. L. Neubarth, T. L. Dickendesher, P. H. Nwe, C. Cassidy, C. J. Woodbury, D. D. Ginty, Genetic identification of an expansive mechanoreceptor sensitive to skin stroking. Cell 163, 1783-1795 (2015).

17. H. Merskey, N. Bogduk, Classification of Chronic Pain. Descriptions of Chronic Pain Syndromes and Definitions of Pain Terms (IASP Press, 1994).

18. S. S. Ranade, S.-H. Woo, A. E. Dubin, R. A. Moshourab, C. Wetzel, M. Petrus, J. Mathur, V. Begay, B. Coste, J. Mainquist, A. J. Wilson, A. G. Francisco, K. Reddy, Z. Qiu, J. N. Wood, G. R. Lewin, A. Patapoutian, Piezo2 is the major transducer of mechanical forces for touch sensation in mice. Nature 516, 121-125 (2014).

19. S.-H. Woo, V. Lukacs, J. C. de Nooij, D. Zaytseva, C. R. Criddle, A. Francisco, T. M. Jessell, K. A. Wilkinson, A. Patapoutian, Piezo2 is the principal mechanotransduction channel for proprioception. Nat. Neurosci. 18, 1756-1762 (2015).

20. A. T. Chesler, M. Szczot, D. Bharucha-Goebel, M. Čeko, S. Donkervoort, C. Laubacher, L. H. Hayes, K. Alter, C. Zampieri, C. Stanley, A. M. Innes, J. K. Mah, C. M. Grosmann, N. Bradley, D. Nguyen, A. R. Foley, C. E. Le Pichon, C. G. Bönnemann, The role of PIEZO2 in human mechanosensation. N. Engl. J. Med. 375, 1355-1364 (2016).

21. S. E. Murthy, M. C. Loud, I. Daou, K. L. Marshall, F. Schwaller, J. Kühnemund, A. G. Francisco, W. T. Keenan, A. E. Dubin, G. R. Lewin, A. Patapoutian, The mechanosensitive ion channel Piezo2 mediates sensitivity to mechanical pain in mice. Sci. Transl. Med. 10, eaat9897 (2018).

22. Å. B. Vallbo, K.-E. Hagbarth, Activity from skin mechanoreceptors recorded percutaneously in awake human subjects. Exp. Neurol. 21, 270-289 (1968).

23. Å. B. Vallbo, H. Olausson, J. Wessberg, N. Kakuda, Receptive field characteristics of tactile units with myelinated afferents in hairy skin of human subjects. J. Physiol. 483, 783-795 (1995).

24. J. Serra, M. Campero, J. Ochoa, H. Bostock, Activity-dependent slowing of conduction differentiates functional subtypes of C fibres innervating human skin. J. Physiol. 515, 799-811 (1999).

25. R. H. Watkins, J. Wessberg, H. B. Wasling, J. P. Dunham, H. Olausson, R. D. Johnson, R. Ackerley, Optimal delineation of single C-tactile and C-nociceptive afferents in humans using latency slowing. J. Neurophysiol. 117, 1608-1614 (2017).

26. R. Ackerley, K. Wiklund Fernstrom, H. Backlund Wasling, R. H. Watkins, R. D. Johnson, Å. B. Vallbo, J. Wessberg, Differential effects of radiant and mechanically applied thermal stimuli on human C-tactile afferent firing patterns. J. Neurophysiol. 120, 1885-1892 (2018).

27. J. Ochoa, H. Torebjörk, Sensation evoked by intraneural microstimulation of single mechanoreceptor units innervating the human hand. J. Physiol. 342, 633-654 (1983).

28. Å. B. Vallbo, K. A. Olsson, K. G. Westberg, F. J. Clark, Microstimulation of single tactile afferents from the human hand. Sensory attributes related to unit type and properties of receptive fields. Brain 107, 727-749 (1984).

29. H. Olausson, J. Cole, K. Rylander, F. McGlone, Y. Lamarre, B. G. Wallin, H. Krämer, J. Wessberg, M. Elam, M. C. Bushnell, Å. Vallbo, Functional role of unmyelinated tactile afferents in human hairy skin: Sympathetic response and perceptual localization. Exp. Brain Res. 184, 135-140 (2008).

30. J. D. Cole, W. L. Merton, G. Barrett, H. A. Katifi, R. D. Treede, Evoked potentials in a subject with a large-fibre sensory neuropathy below the neck. Can. J. Physiol. Pharmacol. 73, 234-245 (1995).

31. J. Cole, M. C. Bushnell, F. McGlone, M. Elam, Y. Lamarre, Å. Vallbo, H. Olausson, Unmyelinated tactile afferents underpin detection of low-force monofilaments. Muscle Nerve 34, 105-107 (2006).

32. J. Minde, O. Svensson, M. Holmberg, G. Solders, G. Toolanen, Orthopedic aspects of familial insensitivity to pain due to a novel nerve growth factor beta mutation. Acta Orthop. 77, 198-202 (2006).

33. J. Minde, T. Andersson, M. Fulford, M. Aguirre, I. Nennesmo, I. N. Remahl, O. Svensson, M. Holmberg, G. Toolanen, G. Solders, A novel NGFB point mutation: A phenotype study of heterozygous patients. J. Neurol. Neurosurg. Psychiatry 80, 188-195 (2009).

34. I. Morrison, L. S. Löken, J. Minde, J. Wessberg, I. Perini, I. Nennesmo, H. Olausson, Reduced C-afferent fibre density affects perceived pleasantness and empathy for touch. Brain 134, 1116-1126 (2011).

35. M. Szczot, J. Liljencrantz, N. Ghitani, A. Barik, R. Lam, J. H. Thompson, D. Bharucha-Goebel, D. Saade, A. Necaise, S. Donkervoort, A. R. Foley, T. Gordon, L. Case, M. C. Bushnell,
C. G. Bönnemann, A. T. Chesler, PIEZO2 mediates injury-induced tactile pain in mice and humans. Sci. Transl. Med. 10, eaat9892 (2018).

36. E. R. Perl, Myelinated afferent fibres innervating the primate skin and their response to noxious stimuli. J. Physiol. 197, 593-615 (1968).

37. Å. B. Vallbo, H. Olausson, J. Wessberg, Unmyelinated afferents constitute a second system coding tactile stimuli of the human hairy skin. J. Neurophysiol. 81, 2753-2763 (1999).

38. S. N. Lawson, Phenotype and function of somatic primary afferent nociceptive neurones with C-, A $\delta$ - or A $\alpha / \beta$-fibres. Exp. Physiol. 87, 239-244 (2002).

39. H. Adriaensen, J. Gybels, H. O. Handwerker, J. Van Hees, Response properties of thin myelinated (A-delta) fibers in human skin nerves. J. Neurophysiol. 49, 111-122 (1983).

40. N. Ghitani, A. Barik, M. Szczot, J. H. Thompson, C. Li, C. E. Le Pichon, M. J. Krashes, A. T. Chesler, Specialized mechanosensory nociceptors mediating rapid responses to hair pull. Neuron 95, 944-954.e4 (2017).

41. A. Arcourt, L. Gorham, R. Dhandapani, V. Prato, F. J. Taberner, H. Wende, V. Gangadharan, C. Birchmeier, P. A. Heppenstall, S. G. Lechner, Touch receptor-derived sensory information alleviates acute pain signaling and fine-tunes nociceptive reflex coordination. Neuron 93, 179-193 (2017).

42. P. C. Garell, S. L. McGillis, J. D. Greenspan, Mechanical response properties of nociceptors innervating feline hairy skin. J. Neurophysiol. 75, 1177-1189 (1996).

43. R. M. Slugg, R. A. Meyer, J. N. Campbell, Response of cutaneous A- and C-fiber nociceptors in the monkey to controlled-force stimuli. J. Neurophysiol. 83, 2179-2191 (2000).

44. C. Krarup, W. Trojaborg, Compound sensory action potentials evoked by tactile and by electrical stimulation in normal median and sural nerves. Muscle Nerve 17, 733-740 (1994).

45. N. Kakuda, Conduction velocity of low-threshold mechanoreceptive afferent fibers in the glabrous and hairy skin of human hands measured with microneurography and spike-triggered averaging. Neurosci. Res. 15, 179-188 (1992).

46. G. Macefield, S. C. Gandevia, D. Burke, Conduction velocities of muscle and cutaneous afferents in the upper and lower limbs of human subjects. Brain 112, 1519-1532 (1989).

47. D. A. Simone, P. Marchettini, G. Caputi, J. L. Ochoa, Identification of muscle afferents subserving sensation of deep pain in humans. J. Neurophysiol. 72, 883-889 (1994).

48. K. Inui, T. D. Tran, M. Hoshiyama, R. Kakigi, Preferential stimulation of A $\delta$ fibers by intra-epidermal needle electrode in humans. Pain 96, 247-252 (2002).

49. T. D. Tran, K. Lam, M. Hoshiyama, R. Kakigi, A new method for measuring the conduction velocities of $A \beta-, A \delta$ - and $C$-fibers following electric and $\mathrm{CO}_{2}$ laser stimulation in humans. Neurosci. Lett. 301, 187-190 (2001).

50. G. D. lannetti, U. Baumgärtner, I. Tracey, R. D. Treede, W. Magerl, Pinprick-evoked brain potentials: A novel tool to assess central sensitization of nociceptive pathways in humans. J. Neurophysiol. 110, 1107-1116 (2013).

51. R. Melzack, P. D. Wall, Pain mechanisms: A new theory. Science 150, 971-978 (1965).

52. R. Richardson, J. Arbit, E. Siqueira, R. Zagar, Transcutaneous electrical neurostimulation in functional pain. Spine 6, 185-188 (1981).

53. C. J. Woodbury, F. A. Kullmann, S. L. Mcllwrath, H. R. Koerber, Identity of myelinated cutaneous sensory neurons projecting to nocireceptive laminae following nerve injury in adult mice. J. Comp. Neurol. 508, 500-509 (2008).

54. B. B. Edin, Quantitative analysis of static strain sensitivity in human mechanoreceptors from hairy skin. J. Neurophysiol. 67, 1105-1113 (1992).

55. M. Trulsson, S. T. Francis, E. F. Kelly, G. Westling, R. Bowtell, F. McGlone, Cortical responses to single mechanoreceptive afferent microstimulation revealed with $\mathrm{fMRI}$. Neuroimage 13, 613-622 (2001)

56. R. M. S. Panchuelo, R. Ackerley, P. M. Glover, R. W. Bowtell, J. Wessberg, S. T. Francis, F. McGlone, Mapping quantal touch using 7 Tesla functional magnetic resonance imaging and single-unit intraneural microstimulation. eLife 5, e12812 (2016).

57. A. B. Sterman, H. H. Schaumburg, A. K. Asbury, The acute sensory neuronopathy syndrome: A distinct clinical entity. Ann. Neurol. 7, 354-358 (1980).

58. W. H. Herman, R. Pop-Busui, B. H. Braffett, C. L. Martin, P. A. Cleary, J. W. Albers, E. L. Feldman; DCCT/EDIC Research Group, Use of the Michigan neuropathy screening instrument as a measure of distal symmetrical peripheral neuropathy in type 1 diabetes: Results from the diabetes control and complications trial/epidemiology of diabetes interventions and complications. Diabet. Med. 29, 937-944 (2012).

59. B. B. Edin, P. A. Bäckström, L. O. Bäckström, Single unit retrieval in microneurography: A microprocessor-based device controlled by an operator. J. Neurosci. Methods 24, 137-144 (1988).

Acknowledgments: We thank L. Koppel, A. Kusztor, and R. Liif for recruitment of participants and patients for their involvement. We thank the NCCIH clinical staff team and A. Necaise for arranging the participation of patients with $A \beta$ deafferentation (GL) and PIEZO $2^{\text {LOF }}$. We thank B. Wang for technical support and W. Jung and R. Liif for assistance in some experiments. We thank G. Westling and A. Bäckström for the development of electronic monofilaments. We are grateful to J. Wessberg, M. Heilig, and D. Mahns for reviewing an earlier version of the manuscript and offering advice. Funding: This work was supported by the Swedish Research Council (H.O.), the ALF Region Östergötland (H.O. and S.S.N.), the Pain Relief Foundation (F.M., A.G.M., 
and F.O.), the Intramural Research Program of the $\mathrm{NIH}$, specifically the NCCIH (A.T.C. and M.C.B.), the DDIR Innovation Award (A.T.C.), and the NINDS (C.G.B.). The PhD stipend of A.M. was funded by the Pain Relief Foundation. Author contributions: H.O., S.S.N., and A.G.M. developed the experimental protocol with input from F.M. and M.T. S.S.N., A.G.M., E.J., and A.M. performed the microneurography experiments with assistance from S.S. and F.O. S.S.N. performed the psychophysical experiments in healthy participants. J.L. performed the psychophysical testing in patients with $A \beta$ deafferentation $(G L)$ and PIEZO2 ${ }^{\text {LOF }}$ with input from M.C.B., A.T.C., D.S., S.D., A.R.F., and C.G.B. S.S.N. performed the psychophysical testing in patients with $A \beta$ deafferentation (IW) and HSAN-V with input from J.C., J.M., and M.R. S.S.N., E.J., and A.G.M. analyzed the electrophysiological data. S.S.N and E.J. produced the figures. S.S.N. wrote the paper with input from all other authors. Competing interests: The authors declare that they have no competing interests. Data and materials availability: All data needed to evaluate the conclusions in the paper are present in the paper and/or the Supplementary Materials. Additional data related to this paper may be requested from the authors.

Submitted 5 December 2018

Accepted 23 May 2019

Published 3 July 2019

10.1126/sciadv.aaw1297

Citation: S. S. Nagi, A. G. Marshall, A. Makdani, E. Jarocka, J. Liljencrantz, M. Ridderström, S. Shaikh, F. O'Neill, D. Saade, S. Donkervoort, A. R. Foley, J. Minde, M. Trulsson, J. Cole, C. G. Bönnemann, A. T. Chesler, M. C. Bushnell, F. McGlone, H. Olausson, An ultrafast system for signaling mechanical pain in human skin. Sci. Adv. 5, eaaw1297 (2019). 


\section{ScienceAdvances}

\section{An ultrafast system for signaling mechanical pain in human skin}

Saad S. Nagi, Andrew G. Marshall, Adarsh Makdani, Ewa Jarocka, Jaquette Liljencrantz, Mikael Ridderström, Sumaiya Shaikh, Francis O'Neill, Dimah Saade, Sandra Donkervoort, A. Reghan Foley, Jan Minde, Mats Trulsson, Jonathan Cole, Carsten G. Bönnemann, Alexander T. Chesler, M. Catherine Bushnell, Francis McGlone and Håkan Olausson

Sci Adv 5 (7), eaaw1297.

DOI: $10.1126 /$ sciadv.aaw1297

ARTICLE TOOLS

SUPPLEMENTARY

MATERIALS

REFERENCES

PERMISSIONS http://advances.sciencemag.org/content/5/7/eaaw1297

http://advances.sciencemag.org/content/suppl/2019/07/01/5.7.eaaw1297.DC1

This article cites 58 articles, 5 of which you can access for free http://advances.sciencemag.org/content/5/7/eaaw1297\#BIBL

http://www.sciencemag.org/help/reprints-and-permissions

Science Advances (ISSN 2375-2548) is published by the American Association for the Advancement of Science, 1200 New York Avenue NW, Washington, DC 20005. The title Science Advances is a registered trademark of AAAS.

Copyright @ 2019 The Authors, some rights reserved; exclusive licensee American Association for the Advancement of Science. No claim to original U.S. Government Works. Distributed under a Creative Commons Attribution NonCommercial License 4.0 (CC BY-NC). 